\title{
ON 3-DIMENSIONAL MANIFOLDS
}

\author{
C. E. CLARK
}

Let $P$ be a 3-dimensional manifold. ${ }^{1}$ Let $Q$ be a 2-dimensional manifold imbedded in $P$. Moreover, let $P$ and $Q$ admit of a permissible simplicial division $K$, that is, a simplicial division of $P$ such that some subcomplex of $K$, say $L$, is a simplicial division of $Q$. Let $K_{i}$ and $L_{i}$ denote the $i$ th normal subdivisions of $K$ and $L$, respectively. We define the neighborhood $N_{i}$ of $L_{i}$ to be the simplicial complex consisting of the simplexes of $K_{i}$ that have at least one vertex in $L_{i}$ together with the sides of all such simplexes. By the boundary $B_{i}$ of $N_{i}$ we mean the simplicial complex consisting of the simplexes of $N_{i}$ that have no vertex in $L_{i}$. Our purpose is to prove the following theorem.

THEOREM. The boundary $B_{2}$ is a two-fold but not necessarily connected covering of $Q$, and change of permissible division $K$ replaces $B_{2}$ by a homeomorph of itself.

Proof. The neighborhood $N_{1}$ is the sum of a set of 3-dimensional simplexes. Some of these 3 -simplexes, say $a_{1}, a_{2}, \cdots$, have exactly one vertex in $L_{1}$, others, say $b_{1}, b_{2}, \cdots$, have exactly two vertices in $L_{1}$, while the remaining, say $c_{1}, c_{2}, \cdots$, have three vertices in $L_{1}$. Since $K_{1}$ is a normal subdivision of $K$, the intersection of $L_{1}$ and $b_{i}$ or $c_{i}$ is a 1 -simplex or 2 -simplex, respectively. Let $\alpha_{i}, \beta_{i}$, and $\gamma_{i}$ be the intersections of $B_{2}$ and $a_{i}, b_{i}$, and $c_{i}$, respectively. We shall regard $\alpha_{i}$ and $\gamma_{i}$ as triangles with vertices on the 1 -simplexes of $a_{i}$ and $c_{i}$. Also we shall regard $\beta_{i}$ as a square with vertices on the 1 -simplexes of $b_{i}$.

Any 2-simplex of $L_{1}$, say $A B C$, is incident to exactly two of the $c_{i}$. Let $c_{1}=A B C M$. There is a unique 3 -simplex of $N_{1}$, say $\sigma$, that is incident to $A B M$ and different from $c_{1}$. This $\sigma$ is either a $c_{i}$, say $c_{2}$, or a $b_{i}$, say $b_{2}$. If $\sigma$ is $c_{2}$, then the triangles $\gamma_{1}$ and $\gamma_{2}$ have a common side. Suppose that $\sigma$ is $b_{2}=A B M N$. The 2 -simplex $A B N$ is incident to a unique 3 -simplex of $N_{1}$, say $\tau$, with $\tau \neq A B M N$. This $\tau$ is either $c_{3}$ or $b_{3}$. If $\tau=b_{3}$, there is a $c_{4}$, or $b_{4}$. Finally we must find a $c_{p}=A B D S, D$ in $L_{1}$, $S$ in $B_{1}$. We now consider $\beta_{2}, \beta_{3}, \cdots$, and $\beta_{p-1}$. The sum of these squares is topologically equivalent to a square. One side of the square is coincident with a side of $\gamma_{1}$ and the opposite side coincident with a side of $\gamma_{p}$.

Received by the editors July 21,1941 .

${ }^{1}$ Our terminology is that of Seifert-Threlfall, Lehrbuch der Topologie. Manifolds are finite, while simplexes and cells are closed point sets. 
Since $K_{1}$ is a manifold, we can repeat the construction and associate with $A B C$ and $A B D$ a second pair of triangles in $B_{2}$ that are either incident along a common side or incident to opposite sides of a square. But there is not a third such configuration associated with $A B C$ and $A B D$. We repeat the construction for all pairs of adjacent 2 -simplexes of $L_{1}$. Then to each 2 -simplex of $L_{1}$ there correspond two triangles in $B_{2}$. Moreover, if two 2 -simplexes of $L_{1}$ are incident along a side, the four corresponding triangles can be paired so that the two triangles of each pair either have a common side or are incident to opposite sides of a square.

Since $P$ and $Q$ are 3 - and 2-manifolds, respectively, we can say that $Q$ is two-sided in $P$ in the neighborhood of any point of $Q$. Moreover, the two $\gamma$ 's of $B_{2}$ that correspond to a 2 -simplex of $L_{1}$ lie on opposite sides of $Q$ (in the neighborhood of this 2-simplex).

Consider a vertex $X$ of $L_{1}$ and the 2 -simplexes $\Delta_{i}$ of $L_{1}$ that have $X$ as a vertex. On one side of $Q$ (in the neighborhood of $X$ ) there corresponds to each $\Delta_{i}$ a unique $\gamma_{i}$, and the $\gamma$ 's have the same incidences as the corresponding $\Delta$ 's (we say that two $\gamma$ 's are incident if they are incident to opposite sides of a square). Let us denote by $R$ the points of these $\gamma$ 's and the squares incident to pairs of these $\gamma$ 's. Let $A$ denote the points of all $\alpha_{i}$ 's that are in $a_{i}$ 's incident to $X$ and on the side of $Q$ that we are considering.

We shall show that $R+A$ is a 2 -cell. To do this we shall show that $R+A$ is a manifold relative to its boundary, that its boundary consists of one or more circles, and that any 1-cycle of $R+A$ bounds in $R+A$. First we observe that $B_{2}$ is a manifold; this fact follows from the structure of $B_{2}$ and the fact that $K_{1}$ is a manifold; the argument is elementary and we omit it. Since $R+A$ is the sum of 2 -cells $\alpha, \beta$, and $\gamma$, the set $R+A$ is a manifold relative to its boundary.

To show that this boundary of $R+A$ consists of one or more circles we shall study the incidences among the cells of $R+A$. First, let $a_{i}$ have $X$ as a vertex. If a 2-dimensional side of $a_{i}$ is not in $B_{1}$, this side must be a side of an $a_{j}$ or $b_{j}$. Furthermore, this $a_{j}$ or $b_{j}$ has $X$ as a vertex. Hence, any side of an $\alpha_{i}$ is also a side of an $\alpha_{j}$ or $\beta_{j}$ of $R+A$. Next, let $c_{i}$ have vertices $X A B M, M$ in $B_{1}$. The sides of $\gamma_{i}$ that are in $X A M$ and $X B M$ are sides of $\gamma_{j}^{\prime}$ 's or $\beta_{j}$ 's of $R+A$. But the side of $\gamma_{i}$ in $A B M$ is not incident to any other 2-cell of $R+A$. This side is part of the boundary of $R+A$. Finally, let $b_{i}$ have vertices $X A M N, A$ in $L_{1}$. The sides of $\beta_{i}$ in $X A M$ and $X A N$ are incident to sides of $\beta_{j}$ 's or $\gamma_{j}$ 's of $R+A$; the side of $\beta_{i}$ in $X M N$ is incident to an $\alpha_{j}$ or $\beta_{j}$ of $R+A$; but the side of $\beta_{i}$ in $A M N$ is not incident to any other 2-cell of $R+A$. This side is part of the boundary of $R+A$. Examination of 
the segments of the boundary of $R+A$ shows that they fit together to form one or more circles.

We next show that if $C$ is a 1 -dimensional cycle of $R+A$, then $C$ bounds in $R+A$. We shall find it convenient to replace $A$ by a new set that will never be empty. We define $A^{\prime}$ to be $A$ together with all vertices of $\gamma$ 's of $R$ that are not in the boundary of $R+A$ and all sides of squares of $R$ that are not sides of $\gamma^{\prime}$ 's of $R$ and not in the boundary of $R+A$. If $A$ is not empty, the set $A^{\prime}$ is the same as $A$. But in any case $A^{\prime}$ is not empty, and $R+A^{\prime}$ is the same set as $R+A$. The set $\left(R+A^{\prime}\right)-\bar{A}^{\prime}$ is homeomorphic to a 2 -cell with an inner point removed because $\left(R+A^{\prime}\right)-\bar{A}^{\prime}$ can be obtained from the configuration of the 2 -simplexes of $L_{1}$ that have $X$ as a vertex by removing $X$ and replacing some of the 1 -simplexes by squares (open along one side). Hence, the cycle $C$ is homologous in $R+A^{\prime}$ to a cycle on $A^{\prime}$, and we assume that $C$ is on $A^{\prime}$. The set $A^{\prime}$ is part of $b$, the boundary of the combinatorial neighborhood of $X$ in $K_{2}$. Since $K_{2}$ is a manifold, the set $b$ is a 2-sphere. Assume that $C$ does not bound in $A^{\prime}$. Then $C$ must surround a 2 -simplex of $b$ that is not in $A^{\prime}$. We easily find a 2 -simplex of $R+A^{\prime}$ that is not incident along one of its sides to another 2-simplex of the manifold $B_{2}$. This contradiction proves that $C$ bounds, and the proof that $R+A$ is a 2 -cell is complete.

Now we draw some lines on $R+A$. If two $\gamma$ 's have a common side, we draw a line coincident with this common side. If two $\gamma$ 's are incident to a square, we draw a line across the square half way between the $\gamma$ 's. All these lines are continued so that they meet at a point of $A$. These lines give a subdivision of $R+A$ that is combinatorially equivalent to the combinatorial neighborhood of $X$ in $L_{1}$. The lines can be drawn for all $R+A$ of $B_{2}$ and we get a subdivision of $B_{2}$ that is combinatorially equivalent to a two-fold but not necessarily connected covering of $L_{1}$.

A triangle of the covering is associated with a 2 -simplex of $L_{1}$ and a side of $Q$ (in the neighborhood of this simplex). Hence, a homeomorphism is determined between this covering and any covering obtained by changing the permissible division $K$.

The theorem is not true with $B_{1}$ rather than $B_{2}$. For example, let $Q$ be the boundary of a 3 -simplex of $K$. Then $B_{1}$ is a sphere and a point.

We can prove the following theorem in the same way but with much less effort.

THEOREM. The above theorem is true if $P$ and $Q$ are replaced by 2 - and 1-dimensional manifolds.

Purdue University 